\title{
Helical Antenna for GPS Applications
}

\author{
Cheng-Wei Lan*, Tze-Hsuan Chang, and Jean-Fu Kiang \\ Department of Eelectrical Engineering and \\ Graduate Institute of Communication Engineering \\ National Taiwan University, Taipei, Taiwan, ROC \\ E-mail: jfkiang@cc.ee.ntu.edu.tw
}

Abstract - Dual-band receiver can be used in global positioning system (GPS) to cancel the effects of ionospheric perturbation. Moreover, increasing the number of satellites may reduce the positioning time and increase the reliability of GPS. In this work, we introduce a bifilar helical antenna which can support both GPS and GLONASS systems.

\section{Introduction}

Helical antenna is one of the most commonly used antenna for satellite communications because of the circular polarization and omnidirectional radiation pattern. Furthermore, fractional-turn quadrifilar helical antenna (QHA) is a compact size antenna which is suitable in mobile handset for global positioning system (GPS) applications.

A theoretical stady of the sheath helix antenna with a conducting core is derived based on the complex solutions of an associated determinantal equation, which provides clues for better understanding of the helical antenna [1]. The solutions of sheath helix model are used in this work to obtain new design of bifilar helical antenna (BHA) which can cover a wide frequency range, including two satellite positioning systems, GPS and GLONASS.

\section{Traveling-Wave Theory for Helical Antenna}

A quadrifilar helical antenna can support both traveling-wave type and resonant type of operation, determined by the element length. The traveling-wave type of operation will be discussed in this section.

\subsection{Unifilar, Bifilar, Quadrifilar Helical Antenna}

Fig.1 shows three-dimensional multifilar helix structures which may be either left or right handed. Parameters to describe a helix structure include helix diameter(D), helix radius(a) circumference(C), spacing between turns $(S)$, pitch angle $(\alpha)$, element length( $L)$, and number of turns(n). The main parameters determine the properties of multifilar helical antenna are pitch angle( $\alpha)$, circumference( $/ \lambda)$, number of turns(n), and element length $(L)$. A subscript $\lambda$ indicates that the parameters are measured in terms of free-space wavelengths. Fig.l(a) shows a conventional unifilar helical antenna. Fig.1(b) shows a bifilar helical antenna(BHA). The BHA is composed of two tape helices equally spaced circumferentially on a cylinder, and fed with equal amplitude signals with relative phase of $0^{\circ}$ and $180^{\circ}$. Fig.l(c) shows a quadrifilar helical antenna (QHA) which is composed of two bifilar helices equally spaced circumferentially on a cylinder and fed with equal amplitude signals with relative phase of $0^{\circ}, 90^{\circ}, 180^{\circ}$, and $270^{\circ}$.

Operation of a QHA is similar in some respects to an unifilar helix antenna. The determinantal equation of $\mathrm{N}$-filar tape helix, excited in the 
$\exp (-\mathrm{j} \phi)$ mode, becomes identical to the sheath helix as the number, $\mathrm{N}$, approaches infinity [2]. Assume that the BHA has the same characteristics with the QHA. Thus, we can estimate the properties of traveling-wave type QHA and BHA by the theory of unifilar helix.

\section{$2.2 k-\beta$ Diagram of Helix Structure}

Periodic structure approach can be applied to analyze the helix of infinite extent by the mode diagram shown in Fig.2. Three modes of operation are marked as modes a, b and c, representing the normal, scanning(back-fire), and axial modes, respectively.

In curve $a$, the cylindrical harmonic of $m=0$ dominates. The axial mode is dominated by the right-handed cylindrical harmonic of $m=1$, also referred to as the $m=1$ supermode.

\subsection{Sheath Helix Model}

Fig.3(a) shows an infinitely long tape helix. Fig.3(b) shows the associated sheath helix model [1]. The helix is modeled as a sheath with different conductivities in different directions. The sheath helix model for an infinitely long helix is mathematically simple but physically inaccurate. Taking the limit that the inner radius, $b$, approaches the outer radius, $a$, we obtain the following four uncoupled solutions

$$
\beta= \pm \mathrm{k}
$$

$$
\beta \mathrm{a} \tan \alpha= \pm \mathrm{ka} / \cos (\alpha)-\mathrm{m}, \mathrm{m}=0, \pm 1 \ldots \ldots
$$

where $m$ indicates the spatial harmonic. The axial propagation constant, $\beta$, can be calculated by solving for the roots of the determinantal equation. The $k-\beta$ diagram of sheath helix is generated by solving $\beta$ with free-space wave number (frequency). The four uncoupled solutions can be used to predict the operation of a helical antenna.

Fig. 4 shows the $k-\beta$ diagram of sheath helix model. At low frequency, the dispersion curve lies within the bound-wave region, the total field is completely bound to the structure and all spatial harmonics are axially slow. When frequency is increased, the $m=1$ harmonic enters the fast-wave region at A1. At this frequency, only one point is located in the fast-wave region, which exhibits a single beam at the back-fire direction in the visible region.

With the frequency increased, the $m=1$ beam scans through the broadside at B1. Subsequently, the beam resulting from $m=1$ harmonic continues to sweep toward the end-fire at El, and then the $m=1$ harmonic leaves the fast-wave region. In the fast-wave region, the wave is a leaky wave. For frequency above $\mathrm{E} 1$, the $\mathrm{m}=1$ harmonic becomes a slow wave and the beam moves into the invisible region.

The $m=2$ harmonic has the same characteristics. For large pitch angle, there are two dispersion curves, $m=1$ and $m=2$, at a given frequency. We should avoid such circumstance when designing helical antenna.

For unifilar helical antenna, the maximum pitch angle is approximately $20^{\circ}$. Reference [8] shows the relation between pitch angle and the number of helices. The optimal pitch angle of BHA is approximately two times that of unifilar HA.

The four uncoupled solutions can also used to obtain the bandwidth of helical antenna and the limit of pitch angle. This result is in good agreement with the experiment results of J. D. Kraus [5].

\section{Antenna Design}

For GPS application, a dual-band receiver can cancel the effects of 
ionospheric perturbation. Moreover, increasing the number of satellites can reduce the positioning time and increase the reliability of the GPS. Currently, there are two major positioning systems, GPS and GLONASS. Various manufacturers of GPS equipments use different size and construction to cover either of the two GPS frequencies at $1575.42 \mathrm{MHz}(\mathrm{L1})$ and $1227.6 \mathrm{MHz}(\mathrm{L}, 2)$. In this work, we design a BHA to cover the four frequencies of GPS and GLONASS.

\subsection{Requirements on GPS Antenna}

For GPS applications, the antenna requires omnidirectional pattern over approximately the entire upper hemisphere where the satellites may be visible with right-handed circular polarization. The wide beamwidth coverage allows the receiver to track as many of the visible satellites as possible. The specifications on GPS and GLONASS antenna include that the frequency range is from $1.2 \mathrm{GHz}$ to $1.65 \mathrm{GHz}$ and the polarization is right handed circular polarization, the axial ratio is $3 \mathrm{~dB}$ maximum at the zenith, and the gain is 4dBi minimum at the zenith.

\subsection{Bifilar Helical Antenna}

Fig. 1(b) shows the design antenna. The pitch angle is $30^{\circ}$. The choice of pitch angle is about two times that of optimal unifilar helical antenna, the minimum $\mathrm{C} / \lambda$ is 0.58 . Considering the mode coupling effect, we make the minimum $\mathrm{C} / \lambda$ to be 0.5 . The lower operation frequency is $1.2 \mathrm{GHz}$, the radius is $21 \mathrm{~mm}$, the number of turns is 3 . The element length which can support the traveling wave operation is $1.38 \lambda$ in $1.2 \mathrm{GHz}$. Fig. 5 (a) shows the gain pattern of right-handed circular polarization. The dashed line is the gain pattem at $1.6 \mathrm{GHz}$, which has the maximum of $7.5 \mathrm{~dB}$, HPBW of $100^{\circ}$, and the front-to-back ratio of $10 \mathrm{~dB}$. The solid line is the gain pattern at $1.2 \mathrm{GHz}$, which has the maximum of $5 \mathrm{~dB}, \mathrm{HPBW}$ of $140^{\circ}$, and the front-to-back ratio of $15 \mathrm{~dB}$.

Fig.5(b) shows the associated axial ratio. The axial ratio at $1.2 \mathrm{GHz}$ has $160^{\circ}$ of beamwidth with $5 \mathrm{~dB}$ maximum. The axial ratio at $1.6 \mathrm{GHz}$ has $174^{\circ}$ of beamwidth with $5 \mathrm{~dB}$ maximum.

Fig.5(c) shows the return loss of the BHA. The input impedance is approximately $250 \mathrm{ohms}$.

Acknowledgment: This work is sponsored by the National Science Council, ROC, under contract NSC 92-2213-E-002-070.

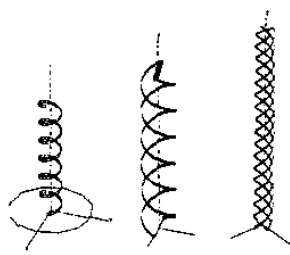

Fig.1: Multi-filar helical antenna, (a) unifilar, (b) bifilar, (c) quadrifilar

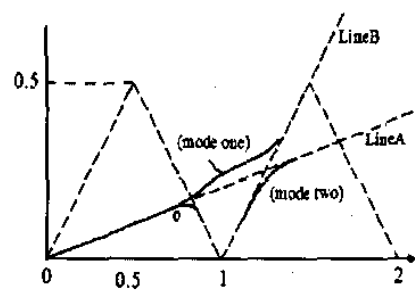

Fig. 2 : $\mathrm{k}-\beta$ diagram of helix structure [4] 


\section{prppr}

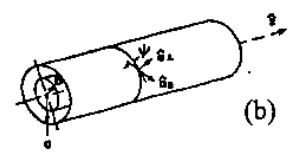

Fig. 3 : Helix model, (a) tape helix model, (b) sheath helix model.

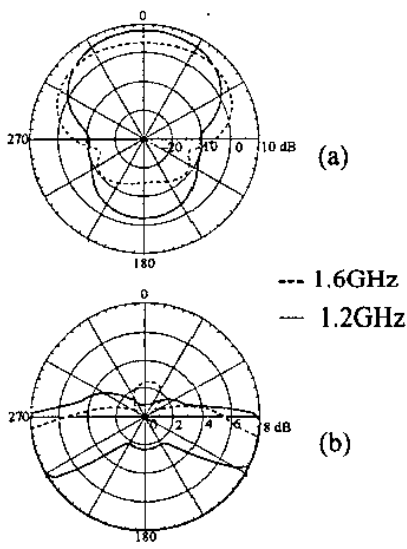

Fig.5: Radiation performance, (a) radiation pattem, (b) axial ratio,

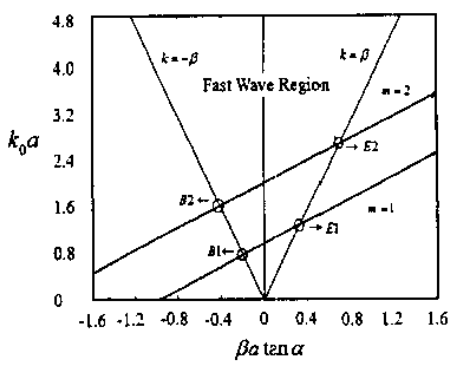

Fig.4: Uncoupling $\mathrm{k}-\beta$ diagram with spatial harmonics $\mathrm{m}=1$ and $\mathrm{m}=2$.

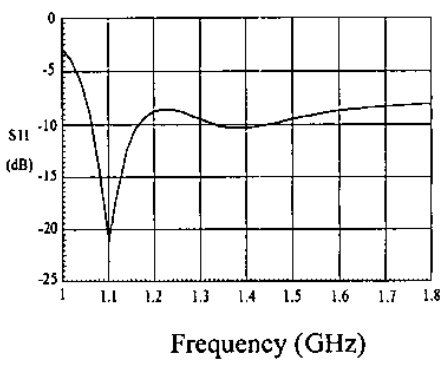

Fig.5(c) : Return loss.

The sheath helix model is used to predict the operation of helical antennas. Design guidelines are based on the uncoupled solution of the sheath helix model. A broadband helical antenna has been introduced which can cover the two existing GPSs.

\section{References}

[1] A,R Neureuther, P.Wklock, and R.Mittra, "A study of sheath helix with a conducting core and its application to the helical anterna," IEEE Trans. Antennas Propagat., vol.15, no.2, Mar. 1967.

[2] C.-H. Ho, L, Fan, and K. Chang, "Ultra wide band slotline hybrid ring couplers," IEEE MTT-S, pp.1175-1178, June 1992.

[3] H. Nakano, "Helical and spiral antennas - a numerical approach," Dept. Elect. Eng. Hosei Univ., Tokyo, Japan.

[4] A. T. Adams, R. K. Greenough, R. F. Wallenberg, A. Mendelovicz, and C. Lumjlak, "The quadrifilar helix antenna", IEEE Trans. Antennas Propagat., vol.22, no.2, Mar. 1974

[5] J. D. Kraus, Antenna, New York: McGraw-Hill, 1950. 